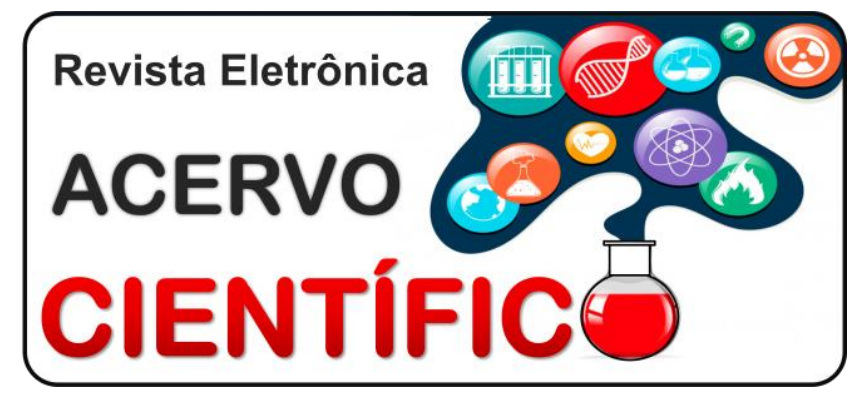

\section{ARTIGO DE REVISÃO}

Recebido em: 7/2019

Aceito em: 7/2019

Publicado em: 8/2019

\title{
A percepção do enfermeiro sobre os cuidados do paciente oncológico terminal exercidos com espiritualidade e religiosidade
}

\author{
The perception of the nurse on the care of terminal cancer patients exercised with \\ spirituality and religiosity
La percepción del enfermero sobre los cuidados del paciente oncológico terminal ejercidos con espiritualidad y religiosidad

Sandra Teresa Figueiredo da Silva ${ }^{1 *}$, Carlos Eduardo Peres Sampaio ${ }^{1-2}$.

\begin{abstract}
Resumo: Identificar os benefícios do cuidado de enfermagem serem exercidos com espiritualidade / religiosidade. A temática foi abordada por meio de revisão integrativa da literatura. Para melhor explicitar os resultados foram construídas quadro categorias, sendo elas: Distinção entre espiritualidade / religiosidade; Benefícios que os cuidados com espiritualidade / religiosidade desempenham na vida do paciente; Dificuldades dos enfermeiros de exercerem os cuidados com espiritualidade/religiosidade; Cuidados de Enfermagem com espiritualidade / religiosidade em meio ao sofrimento espiritual do paciente sem possibilidade terapêutica. Conclui-se o estudo com a premissa que a fé em Deus é o apoio consagrado pela espiritualidade visto que favorece um excelente domínio pessoal diante da circunstância do câncer em fase terminal.
\end{abstract}

Palavras-chave: Espiritualidade, Cuidados de Enfermagem, Enfermagem Oncológica.

Abstract: Identify the benefits of nursing care to be exercised with spirituality / religiosity. The thematic was approached through an integrative review of the literature. To better explain the results, categories were constructed, being: Distinction between spirituality / religiosity; Benefits care of spirituality / religiosity play in the life of the patient; Difficulties of nurses to exercise care with spirituality / religiosity to the patient; Nursing care with spirituality / religiosity in the midst of the spiritual suffering of the patient without therapeutic possibility. The study concludes with the premise that faith in God is the support consecrated by spirituality since it favors an excellent personal domain in the face of the circumstance of cancer in terminal phase.

Keywords: Spirituality, Nursing Care, Oncology Nursing.

Resumen: Identificar los beneficios del cuidado de enfermería para ser ejercitado con espiritualidad / religiosidad. La temática se abordó a través de una revisión integradora de la literatura. Para explicar mejor los resultados, se construyeron categorías, siendo: Distinción entre espiritualidad / religiosidad; Beneficios del cuidado de la espiritualidad / religiosidad en la vida del paciente; Dificultades de las enfermeras para ejercer el cuidado con espiritualidad / religiosidad para el paciente; Cuidados de enfermería con espiritualidad / religiosidad en medio del sufrimiento espiritual del paciente sin posibilidad terapéutica. El estudio concluye con la premisa de que la fe en Dios es el apoyo consagrado por la espiritualidad, ya que favorece un excelente dominio personal ante la circunstancia del cáncer en la fase terminal.

Palabras clave: Espiritualidad, Cuidados de Enfermería, Enfermería Oncológica.

1 Universidade Veiga de Almeida (UVA), Rio de Janeiro - RJ. *E-mail: sandrateresafigueiredo@gmail.com

2 Universidade Estadual do Rio de Janeiro (UERJ), Rio de Janeiro - RJ. 


\section{INTRODUÇÃO}

Cabe esclarecer a evolução sobre o estudo voltado para os cuidados de enfermagem exercidos com espiritualidade e religiosidade, visto que, na prática clínica, o enfermeiro realiza cuidados abrangentes, ou seja, corpo e mente (TAVARES MM et al., 2018). Ressalta-se, no entanto, que ignorar este estudo ou mesmo torná-lo insignificante na prática clínica, seria desconsiderar pesquisas científicas as quais revelam que a crença inabalável em algo, melhora a qualidade de vida e concede forças para o paciente com doença oncológica terminal suportar o tratamento (MENDONÇA AB et al., 2018).

A enfermagem no exercício do cuidado ao paciente oncológico deve ter a espiritualidade como método específico visto que esse cuidado proporciona conforto e tranquilidade aos pacientes no fim de vida. Neste contexto é imprescindível incluir a espiritualidade/religiosidade no currículo da formação acadêmica da equipe interdisciplinar com o propósito de favorecer os profissionais da saúde e de auxiliar os pacientes a desfrutarem um fim de vida livre de angústia espiritual (ARRIEIRA ICO et al., 2016).

Prestar assistência ao paciente com doença oncológica, significa enfrentar complexidades fruto da confirmação da patologia. Diante disso, profissionais de saúde, na prática clínica, analisaram que pacientes e seus familiares tornaram-se mais fortes quando buscam ajuda da espiritualidade para resistir ao choque emocional tão frequentes nessas circunstâncias (JUNIOR RFS et al., 2016).

A espiritualidade melhora a vida das pessoas, visto que desenvolve algo nobre, principalmente, no contexto de uma doença grave como o câncer. Além disso, a espiritualidade encoraja, impulsiona não apenas o paciente ao enfrentar a morte, como também aos profissionais a realizarem os cuidados do paciente à beira da morte (ARRIEIRA ICO et al., 2011). Portanto este estudo teve como objetivo identificar os benefícios dos cuidados de enfermagem serem exercidos com espiritualidade/religiosidade.

\section{MÉTODOS}

Revisão Integrativa de Literatura (RIL), é um método científico que direciona profissionais de diferentes áreas a se atualizarem, através da pesquisa de estudos anteriores para melhor incorporação de evidências na prática clínica para os auxiliarem na melhor maneira de prestar o cuidado e, dessa maneira, melhorar a qualidade de vida do paciente. (MENDES KDS et al., 2008).

Este estudo (RIL) foi realizado fundamentalmente em seis etapas: 1) A construção da pergunta norteadora, a qual foi: "A percepção do enfermeiro sobre os cuidados do paciente oncológico terminal exercidos com espiritualidade / religiosidade"; 2) Busca nas bases de dados eletrônicas - A pesquisa foi realizada no mês de março 2019, em três bases de dados: Literatura Latino Americana e do Caribe (LILACS); Scientífics Electronic Library Online (SciELO); Banco de Dados em Enfermagem (BDENF) na plataforma de dados da Biblioteca Virtual da Saúde (BVS), utilizando o booleano AND e os seguintes descritores: "Enfermagem Oncológica" AND "Espiritualidade" "Cuidados de Enfermagem" AND "Espiritualidade". 3) Seleção da literatura segundo o critério de inclusão e exclusão. Foram incluídos no estudo: artigos originais e completos, elaborados em português e publicados entre 2011 a 2018.

Os critérios de exclusão foram: artigos duplicados, teses, dissertações, livros e artigos que não abordaram o tema da revisão; 4) Análise crítica dos artigos incluídos. Os artigos potencialmente relevantes para a revisão, foram selecionados com base na leitura dos títulos e resumos. Essa seleção seguiu um protocolo elaborado pelos autores e composta por variáveis a fim de assegurar a exatidão da pesquisa. Elegeu-se nesta etapa 14 artigos.

O Fluxograma na (Figura 1) sintetiza a construção do corpus desta revisão 5) Discussão dos resultados - Procedeu-se a análise crítica para justificar a escolha de cada estudo, bem como sua interpretação e síntese dos resultados; 6) Apresentação da revisão. As informações obtidas foram agrupadas e apresentadas em tabela (Tabela 1), presente nos resultados, objetivando a uma melhor visualização dos estudos incluídos nesta revisão integrativa. Desse agrupamento emergiram as categorias e nível de evidência dos estudos. 
Figura 1 - Fluxograma da seleção dos estudos.

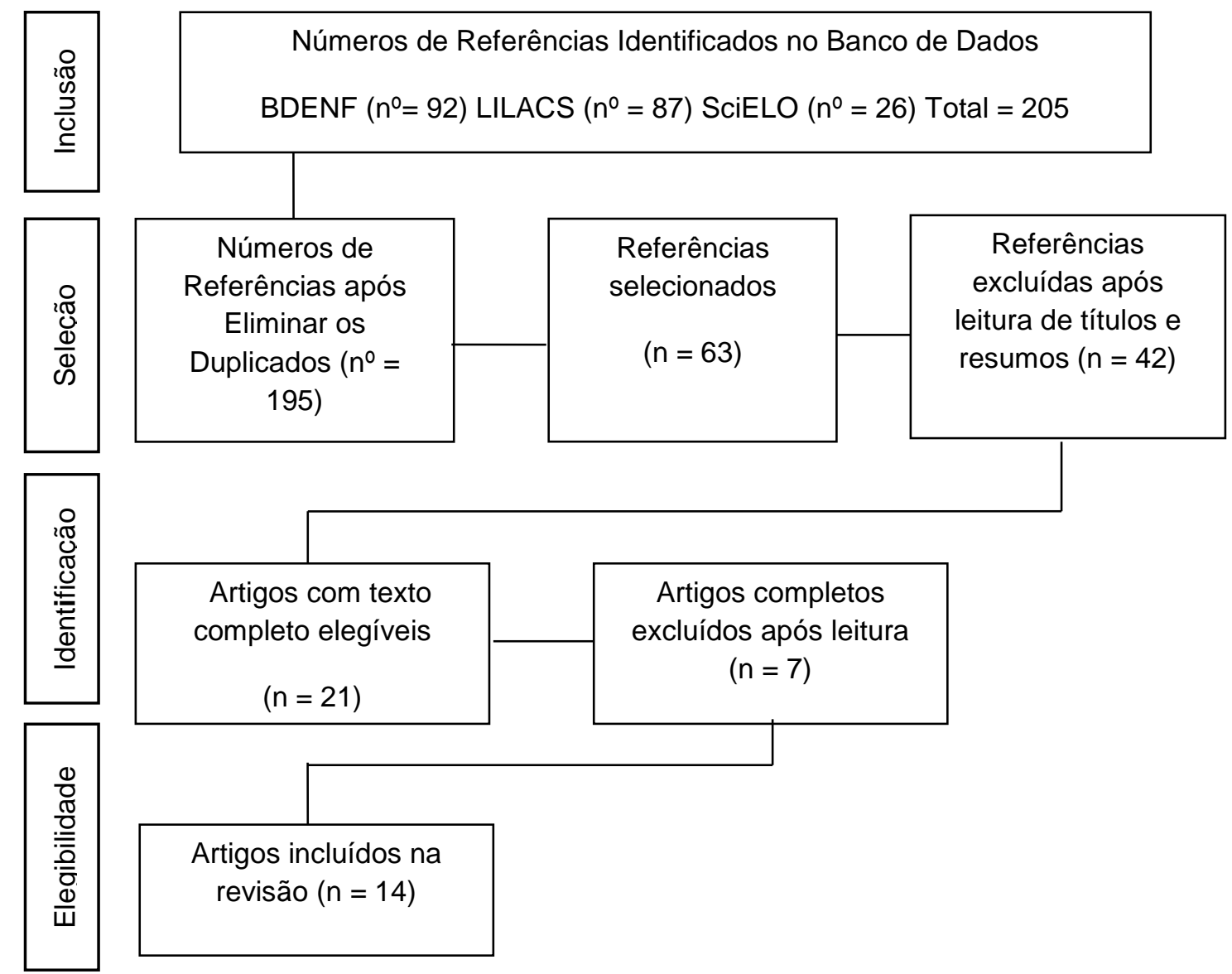

Fonte: Dados da pesquisa, 2018.

\section{RESULTADOS}

Os delineamentos dos 14 artigos eleitos compõem-se em: estudos quantitativos $(n=2)$; estudos qualitativos $(n=9)$; estudos descritivos $(n=1)$ e; estudos de revisão integrativa da literatura $(n=2)$. Observa-se que autores são profissionais enfermeiros e integrantes da equipe interdisciplinar de cuidados paliativos.

No que diz respeito aos intervalos de tempo das publicações, no ano de 2016 , sucedeu elevado número de publicações $(n=5)$. em $2018(n=4), 2017,(n=2)$; 2015, $(n=1)$; 2013, $(n=1)$ e 2011, $(n=1)$.

Os principais resultados relacionados ao objetivo dessa revisão foram arregimentados em quatro categorias, a saber: 1) Distinção entre espiritualidade / religiosidade; 2) Benefícios que os cuidados com espiritualidade / religiosidade desempenham na vida do paciente; 3) Dificuldades dos enfermeiros de exercerem os cuidados com espiritualidade / religiosidade e; 4) Cuidados de Enfermagem com espiritualidade / religiosidade em meio ao sofrimento espiritual do paciente sem possibilidade terapêutica.

Para melhor aprimoramento e entendimento das categorias expostas, viu-se a necessidade de organizálas agrupando-as de acordo com título, nome dos autores, periódicos, ano de publicação, nível de evidência e objetivo da pesquisa (Tabela 1). 
Tabela 1 - Artigos incluídos na revisão integrativa título, nome dos autores, periódicos, ano de publicação, nível de evidência e objetivo da pesquisa. Rio de Janeiro - RJ, 2019.

\begin{tabular}{cl}
\hline Categoria & \multicolumn{1}{c}{ Título, autores, periódico e ano } \\
\hline 1 & Espiritualidade na Equipe interdisciplinar que atua em cuidados paliativos \\
as pessoas com câncer. ARRIEIRA ICO et al. Ver. Cienc. Cuid. Saúde
\end{tabular}

1 as pessoas com câncer. ARRIEIRA ICO et al. Ver. Cienc. Cuid. Saúde (2011).

1 Espiritualidade na iminência da morte estratégia adotada para humanizar o cuidar em enfermagem. BRITO FM et al. Revista Enf. UFRJ (2013)

Influência da Espiritualidade no tratamento do usuário Oncológico: olhar

1 do Enfermeiro. SAMPAIO AD et al. Rede de Revista Cientificas da América Latina. (2016)

Cuidados paliativos e Enfermagem. EVANGELISTA CB et al. Revista

$1 \quad$ Bras. Enferm. REBEN (2015)

\section{Nível de Evidência}

2 Influência da Espiritualidade no tratamento do usuário Oncológico: olhar do Enfermeiro. SAMPAIO AD et al. Rede de Revista Cientificas da América Latina, Caribe, Espanha e Portugal. (2016)

2 Percepção de equipe de enfermagem sobre espiritualidade nos cuidados de final de vida. SILVA BS et al. Revista Cogitare Enferm. (2016)

2 Refletindo sobre a espiritualidade e a religiosidade relacionados a prática dos profissionais de saúde na oncologia. JUNIOR RFS et al. Revista Acervo Saúde (2016)

2 O sentido da espiritualidade na transitoriedade da vida. ARRIEIRA ICO et al. Revista Escola Anna Nery (2017)

2 Espiritualidade e Religiosidade no Cotidiano da enfermagem hospitalar. TAVARES MM et al. Revista Enferm. UFPE (2018)
V

V

V

\section{Objetivo}

Conhecer o significado da espiritualidade para a equipe do Programa de Internação Domiciliar Interdisciplinar Oncológico, que oferece cuidados paliativos.

Investigar a compreensão de enfermeiros sobre conceitos de espiritualidade e de necessidades espirituais do paciente sem possibilidades terapêuticas

Analisar a influência da espiritualidade no tratamento do usuário oncológico sob o olhar do enfermeiro.

Analisar artigos científicos disseminados em periódicos on-line no cenário internacional acerca da temática cuidados paliativos espiritualidade.

Analisar a influência da espiritualidade no tratamento do usuário oncológico sob o olhar do enfermeiro.

Investigação a percepção da equipe de enfermagem acerca da espiritualidade nos cuidados de final de vida.

Compreender a espiritualidade religiosidade como prática para o cuidado em saúde.

Compreender o sentido da espiritualidade para a pessoa em cuidados paliativos.

Promover reflexões sobre a presença da espiritualidade e religiosidade no cotidiano do enfermeiro hospitalar. 
Percepção de equipe de enfermagem sobre espiritualidade nos cuidados de final de vida. SILVA BS et al. Revista Cogitare Enferm. (2016)

Espiritualidade na iminência da morte estratégia adotada para humanizar o cuidar em enfermagem. BRITO FM et al. Revista Enferm UFRJ (2013)

3 O sentido da espiritualidade na transitoriedade da vida. ARRIEIRA ICO et al. Revista Escola Anna Nery (2017)

3 Cuidados paliativos e Enfermagem. EVANGELISTA CB et al. Revista Bras. Enferm. REBEN (2015)

Influência da Espiritualidade no tratamento do usuário Oncológico: Olhar

3 da Enfermagem. SAMPAIO AD et al. Rede de Revista Cientificas da América Latina, Caribe, Espanha e Portugal (2016)

Percepção de equipe de enfermagem sobre espiritualidade nos cuidados

4 de final de vida. SILVA BS et al. Revista Cogitare Enferm. (2016)

$4 \quad$ Espiritualidade na iminência da morte estratégia adotada para humanizar o cuidar em enfermagem. BRITO FM et al. Revista Enferm. UFRJ (2013)

4 O sentido da espiritualidade na transitoriedade da vida. ARRIEIRA ICO et al. Revista Escola Anna Nery (2017)

A espiritualidade e a religiosidade como estratégias de enfrentamento do

4 adoecer e morrer. BEZERRA MSM et al. Revista Cienc. Cuid. Saúde. (2018)

$4 \quad$ O sentido do cuidado espiritual na integralidade da atenção em cuidados paliativos. ARRIEIRA ICO et al. Revista Gaúcha Enferm. (2017)
V
Investigar a percepção da equipe de enfermagem acerca da espiritualidade nos cuidados de final de vida.

Investigar a compreensão de enfermeiros sobre conceitos de espiritualidade e de necessidades espirituais do paciente sem possibilidades terapêuticas.

Compreender o sentido da espiritualidade para a pessoa em cuidados paliativos.

Analisar artigos científicos disseminados em periódicos on-line no cenário internacional acerca da temática cuidados paliativos espiritualidade.

Analisar a influência da espiritualidade no tratamento do usuário oncológico sob o olhar do enfermeiro.

Investigar a percepção da equipe de enfermagem acerca da espiritualidade nos cuidados de final de vida.

Investigar a compreensão de enfermeiros sobre conceitos de espiritualidade e de necessidades espirituais do paciente sem possibilidades terapêuticas.

Compreender o sentido da espiritualidade para a pessoa em cuidados paliativos.

Compreender a espiritualidade e a religiosidade como estratégia de enfrentamento do adoecimento e da morte na juventude.

Compreender o sentido do cuidado espiritual para a integralidade da atenção à pessoa e para a equipe interdisciplinar de cuidados paliativos.

Fonte: Dados da pesquisa, 2018. 


\section{DISCUSSÃO}

\section{Categoria 1 - Distinção entre Espiritualidade e Religiosidade}

A religião é classificada como credo, doutrina, ato solene ou adoração a figura. A espiritualidade não se enquadra na postura catedrática e ideológica, porém tais conceitos teóricos identificam-se já que a religião é o caminho para chegar à espiritualidade (BRITO FM et al., 2013).

A espiritualidade destoa da religiosidade. A religiosidade é compreendida como agrupamento, doutrina, seita ou credo associado a um deus que reúne pessoas para a prática de rituais. A espiritualidade em tese é o oposto de religiosidade, sendo classificada como uma ferramenta considerável no cuidado dos pacientes oncológicos. Logo, apresenta-se significativamente inserida no contato ao paciente de maneira espontânea, a fim de perseverar na procura da razão de viver. Além disso, exerce ação preventiva e age na causa do problema (ARRIEIRA ICO et al., 2011).

A espiritualidade possui múltiplas dimensões que alcança um sentido para a existência do nascimento até a morte. Associa-se a crença no "Onipotente", "Salvador", "Altíssimo e Senhor", "Deus", "Espírito Santo" ou "Natureza Divina", assim, é uma particularidade que faz parte do ser humano. É uma força que faz quem crer se sentir mais forte para amar a si, perdoar a quem o magoou, superando todo desgosto (EVANGELISTA CB et al., 2016).

A espiritualidade e a religiosidade podem estar associadas, no entanto seus significados não são semelhantes. A espiritualidade é conectada a um ser sublime, com quesitos inquestionável sobre significado e a finalidade da existência. A religiosidade abrange um procedimento de liturgia e princípios ditados por um líder religioso (SAMPAIO AD et al., 2016).

\section{Categoria 2 - Benefícios que os cuidados com espiritualidade/religiosidade desempenham na vida do paciente}

A energia que a espiritualidade / religiosidade proporcionam ao paciente $o$ ajuda a suportar a doença e a morte, logo a notificação da patologia passa ser tolerável já que pela fé entregou tudo nas mãos de Deus, entendendo ser essa a sua escolha (SAMPAIO AD et al., 2016).

A doença oncológica provoca muitas emoções negativas no paciente, gerando conflitos como: medo de morrer, arrependimento, raiva, lamentação. Neste contexto a espiritualidade mostra-se relevante subterfúgio para suportar a patologia e minimizar o desânimo impelido pelo diagnóstico (SILVA BS et al., 2016).

Um determinado estudo evidenciou que dez pacientes com câncer responderam que a fé que possuíam em Deus os fortaleceram, esses integrantes conferiram à religiosidade e à espiritualidade a razão do seu restabelecimento e auxílio na terapêutica (JUNIOR RFS et al., 2016).

Por meio da espiritualidade é solucionada as causas da angústia espiritual, uma vez que, segundo estudo, a fé e leitura do livro sagrado, como estratégia de apoio, é um ótimo benefício, assim mediante a patologia a fé é fortalecida (ARRIEIRA ICO et al., 2017).

A espiritualidade e a religiosidade, grandezas que atuam na qualidade de vida do paciente hospitalizado, fortalecem a certeza e a confiança que Deus está no comando. Portanto, para o paciente há manifestação de aprovação e apoio ao tratamento indicado (TAVARES MM et al., 2018).

\section{Categoria 3 - Dificuldades dos enfermeiros de exercerem os cuidados com espiritualidade / religiosidade}

Considera-se que o sofrimento espiritual dos pacientes com patologias de risco de morte, normalmente não é solucionado pelo enfermeiro devido falta de capacitação e treinamento (ARRIEIRA ICO et al., 2017).

A enfermagem apresenta dificuldade de exercer o cuidado com espiritualidade, porque o executar e 0 explorar, na história da enfermagem, até então, estão assustadoramente incutidos no paradigma predominante usado por médicos, os quais tratam o paciente de maneira formal e imparcial (SILVA BS et al., 2016). 
Estudos realizados com enfermeiros apontam a incapacidade dos profissionais em atenderem as exigências espirituais / religiosas dos pacientes. Em relação a esse mesmo estudo um outro evidenciou que a insuficiência de assistência dessas exigências espirituais e religiosas podem resultar no paciente desespero, aflição, inquietude impossibilitando o de lutar e assim tornando o prognóstico físico e emocional mais intenso (BRITO FM et al., 2013).

Ainda que os pacientes exteriorizem a sede de ouvir falar, no decorrer da assistência, a Palavra de Deus, mesmo sabendo que essa prática promove a paz e suporte para o paciente enfrentar as consequências do tratamento, existem indícios que expressar sobre a espiritualidade, como parte do cuidado, constantemente é evitado pelo enfermeiro pelo bloqueio de desconhecer o que significa cuidar com espiritualidade, principalmente pela falta de capacitação espiritual nesse âmbito. Estudos ratificam que o empecilho que discorre sobre a espiritualidade, na opinião dos enfermeiros, é o receio de incutir pensamentos religiosos que podem ir de encontro a crença religiosas do paciente (EVANGELISTA CB et al., 2016).

A espiritualidade, deve ser acrescentada ao cuidado holístico, posto que pertence ao ser humano, porém percebe-se condutas tímidas por partes dos enfermeiros em prestar cuidado espiritual. De acordo com estudos anteriores, os enfermeiros, ao serem questionados sobre o assunto, declaram que durante a graduação não foram habilitados para dar conta das demandas espirituais dos pacientes (SAMPAIO AD et al., 2016).

\section{Categoria 4 - Cuidados de Enfermagem com Espiritualidade / Religiosidade em meio ao Sofrimento Espiritual do Paciente sem Possibilidade Terapêutica}

As atitudes espirituais e religiosas atuam mutuamente com a alteração da saúde, em especial em contexto de enfermidade tão preocupante como o tumor maligno. Tal patologia produz mudanças na pessoa investida, especialmente, na iminência do falecimento. Nesse caso, a fé e a espiritualidade são subterfúgios revelados conivente por provocar alívio no período mais complexo da doença. Em suma explorar a espiritualidade na prática clínica faz-se determinante nos instantes que se estar próximo de quem vai partir (BEZERRA MSM et al., 2018).

Estudos revelam que a espiritualidade possibilita expectativa na saúde física e emocional, quando o paciente está no final da vida, amenizando angústia, reduzindo o estresse e descrença. Desenvolvendo um estado de equilíbrio na despedida da vida (BRITO FM et al., 2013).

Estudos evidenciam a iminência da equipe de enfermagem concordarem com os benefícios que os cuidados com espiritualidade realizam na vida do paciente terminal. Cuidados baseados em ouvir atentamente o paciente, aprovar as atividades espirituais do paciente e familiares encorajando-os a se respaldarem na fé, desde que a prática não influencie, negativamente, no tratamento. Nesse contexto cooperar com um final de vida honrado, com cuidados abrangente e menos angustiante (SILVA BS et al., 2016).

A fé é o amparo assinalado pela espiritualidade que beneficia o paciente diante da doença oncológica terminal. Acredita-se que as religiões concedem a respostas sobre o impasse diante do fim da vida, normalmente, referente a figura de Deus, independentemente, da religiosidade da pessoa. Constata-se que paciente no final da vida, o socorro de Deus é muito presente, além disso, vários estudos admitem que no contexto da morte a fé é fortalecida (ARRIEIRA ICO et al., 2017).

\section{CONSIDERAÇÕES FINAIS}

Conclui-se que o cuidado de enfermagem com espiritualidade/religiosidade melhora a qualidade de vida do paciente oncológico concedendo Ihe força para suportar o tratamento. De acordo com as evidências presente neste estudo, espera se que os enfermeiros se conscientizem da importância de ler a Palavra de Deus e de orar com os enfermos durante o cuidado holístico, uma vez que a espiritualidade é inerente ao ser humano. Portanto, é fundamental incluí-la no currículo da formação acadêmica objetivando qualificar os profissionais de saúde para que tenham maior atenção sobre a dimensão espiritual do paciente. 


\section{REFERÊNCIAS}

1. ARRIEIRA ICO, et al. Espiritualidade na equipe interdisciplinar que atua em cuidados paliativos às pessoas com câncer. Ciênc. Cuid. Saúde, v. 10, n.2, p. 314-321, 2011.

2. ARRIEIRA ICO, et al. Espiritualidade e o processo de morrer: reflexões de uma equipe interdisciplinar de cuidados paliativos. Av. enferm., Bogotá, v.34, n.2, p.137-147, 2016.

3. ARRIEIRA ICO, et al. O sentido da espiritualidade na transitoriedade da vida. Esc. Anna Nery, Rio de Janeiro, v. 21, n. 1, e20170012, 2017.

4. ARRIEIRA ICO, et al. O sentido do cuidado espiritual na integralidade da atenção em cuidados paliativos. Revista Gaúcha de Enfermagem. Porto Alegre. 2017; 38(3):e58737.

5. BRITO FM, et al. Espiritualidade na iminência da morte: estratégia adotada para humanizar o cuidar em enfermagem. Revista Enfermagem UERJ. 2014; 21(4): 483-489.

6. BEZERRA MSM, et al. A espiritualidade e a religiosidade como estratégias de enfrentamento do adoecer e morrer. Ciênc Cuid Saude. 2018; 17(4): e 451-55.

7. EVANGELISTA CB, et al. Cuidados paliativos e espiritualidade: revisão integrativa da literatura.Rev. Bras. Enferm. 69(3): 591-6.

8. IENNE A, et al. A espiritualidade de enfermeiros assistenciais interfere no registro do diagnóstico sofrimento espiritual? Esc. Anna Nery, Rio de Janeiro. 2018; 22(1): e20170082.

9. JUNIOR RFS, et al. Refletindo sobre a espiritualidade e a religiosidade relacionada à prática dos profissionais de saúde. Vol. Sup. 4, S178-S185 2016.

10. MENDES KDS, et al. Revisão integrativa: método de pesquisa para a incorporação de evidências na saúde e na enfermagem. Texto Contexto Enferm Florianópolis 2008 17(4)758-64.

11. MENDONCA AB, et al. Aconselhamento e assistência espiritual a pacientes de quimioterapia: uma reflexão à luz da teoria de Jean Watson. Esc. Anna Nery. 2018; 22(4): e20180081.

12. NASCIMENTO LC, et al. Atenção às necessidades espirituais na prática clínica de enfermeiros. Aquichan. Bogotá. 2016; 16(2):179-192.

13. SAMPAIO AD, SIQUEIRA HCH. Influência da Espiritualidade no Tratamento do Usuário Oncológico: Olhar da Enfermagem. Ensaios e Ciência: Ciências Biológicas, Agrárias e da Saúde. 2016; Vol. 20,151-158.

14. SILVA BS, et al. Percepção da equipe de enfermagem sobre espiritualidade nos cuidados de fim de vida. Cogitare enferm.; 21(4):01-07. dez.2016. ISSN 2176-9133.

15. TAVARES MM, et al. Espiritualidade e religiosidade no cotidiano da enfermagem hospitalar. Rev. enferm. UFPE on line. 2018; 12(4):1097-1102. 